\title{
DECISION SUPPORT SYSTEM FOR DISPATCHING PERSONNEL OF INTELLIGENT ELECTRIC POWER SYSTEMS
}

\author{
Mikhail Andreev ${ }^{1, a}$, Almaz Sulaymanov ${ }^{1}$ and Alexey Suvorov ${ }^{1}$ \\ ${ }^{1}$ Institute of Power Engineering, National Research Tomsk Polytechnic University, Lenina Avenue, 30, 634050, \\ Tomsk, Russia
}

\begin{abstract}
This article summarizes the results of an analytical review of usage in Russian and the world information and telecommunications decision support systems for dispatching personnel of electric power systems (ITDSS DP EPS). As the key methods for development of ITDSS DP EPS the numerical and hybrid approaches to electric power system (EPS) simulation were highlighted. Theoretical substantiation of unacceptability of the first variant allowed choosing the hybrid approach as a major which allows developing and applying the most effective methods, ways and means for every significant aspect of the problem of creation of ITDSS DP EPS. In accordance with this approach the most optimal variant of ITDSS DP EPS was developed. The description of this also briefly presented in the article.
\end{abstract}

\section{Introduction}

In our days actively are being developed and integrated the newest microprocessor protection devices, automation and control systems, implemented with using modern IT-technologies and have the ability to adapt to a particular regime of electric power systems (EPS). Their integration helps reduce electrical losses, improve controllability and sustainability of EPS. The "traditional" power systems are transformed into intelligent (IEPS). The objectives of development and research IES is a priority worldwide $[1,2]$. According to the report of the European Commission about Smart Grid projects (Smart Grid Projects Outlook 2014) [3] on the implementation of the projects more than 3 billion Euros is allocated. In the United States - almost 8 billion USD [4]. In total in the world for the development of so-called "super network" and IEPS as a whole last year has been allocated more than 30 billion USD.

Despite the extensive automation, all major decisions on the various changes in schemes and regimes of IEPS are made by dispatching personnel. The task of IEPS' control, taking into account the above-mentioned specifics, is extremely difficult and incorrect or delayed actions can cause serious system failures, which are associated with significant economic and technological damage. Due to it we can conclude that the use of decision support systems (DSS) for dispatching personnel of IEPS is vitally important.

\footnotetext{
${ }^{a}$ Corresponding author : andreevmv@tpu.ru
} 


\section{Analytical reviews of decision support systems for dispatching personnel of power systems}

Analysis of Russian and foreign training simulators, advisers and DSS for dispatchers of IEPS in particular $[5,6]$, led to the conclusion that these systems are use rather simplified mathematical models of IEPS' equipment. Otherwise - the size of total mathematical model of IEPS is significantly reduced. Meanwhile, in real networks of IEPS all interconnected elements (equipment) of IEPS with various sensitivity factors impact the processes and regimes. Therefore, for obtaining sufficiently complete and accurate information about regimes of IEPS, which is necessary for the correct, informed and efficient making decision for control of equipment and IEPS as a whole, helping to reduce the level of accidents in the power system by reducing the number of incorrect and/or belated action of dispatchers, increase "survivability" of electrical power systems DSS, the following should be done:

1 Simulation of equipment and IEPS as a whole using real three-phase scheme of IEPS with its permitted partial equivalenting.

2 Application the three-phase model of all elements of IEPS (generators, including excitation system and prime movers; synchronous and asynchronous electric motors, including their driven mechanisms and excitation system (for synchronous electric motors); transformers; power transmission lines; shunt reactors; condenser batteries; generalized loads and FACTS devices; switching equipment and etc.) for continuously, adequate and reliable simulation of the single spectrum of the quasi-steady-state and transient processes in all possible normal, emergency and postemergency regimes of operation.

3 Providing guaranteed and acceptable accuracy of solutions to mathematical models of IEPS equipment on the unlimited time-interval.

These conditions for the successful solution to the investigated problem and known specifics and complexity of IEPS exclude receiving full information, which is needed for making the correct decisions for the optimal control of IEPS, by experiments in real power system or by means of physical modeling.

As alternative directions and methods, techniques and tools for solving the problem the following methods can be used:

1 Digital simulation of IEPS using only numerical methods, ways and means for calculating the mathematical models.

2 Complex (hybrid) modeling [7-9] of IEPS suggested for achieving the radical solution to various aspects and problems as a whole application, creation and integration of analog, digital-to-analog, digital, analog-to-digital and physical methods, ways and means.

Theoretical substantiation, which is partly reflected in the publications [7-9], of unacceptability of the first variant allowed choosing as the main hybrid approach, which allows developing and applying for every significant aspect of the problem of DSS' creating the most effective methods, ways and means, aggregation of which ensures successful solution to the problem as a whole.

\section{Possible ways of the DSS' development}

The volume of this paper does not allow giving all details of the DSS' development. We can only say that in accordance with the hybrid approach it was created the most optimal variant of the structure of the DSS [further - information and telecommunications decision support systems for dispatching personnel of electric power systems (ITDSS DP EPS)] with the multi-level multi-processor data management system (DMS) (Figure 1) and its software and hardware implementation (Figure 2).

Figure 1: CPU - central processing unit; PP - peripheral processors; HCP - hybrid co-processor; SP - specialized processor; LAN - local area network; SCADA - supervisory control and data acquisition.

Figure 2: NS - network switch; HMLC - hardware module for local connection; HMPC hardware module physical connection; PUC - processor unit for control; PUMMC - processor unit for 
mathematical models calculation; DB - digital bus; CMVV - continuous mathematical variables of voltages; CMVC - continuous mathematical variables of currents; UPU - universal processor unit; $\mathrm{ABC}$ - three-phase inputs/outputs; TPS - three-phase switch.

LEVEL 4

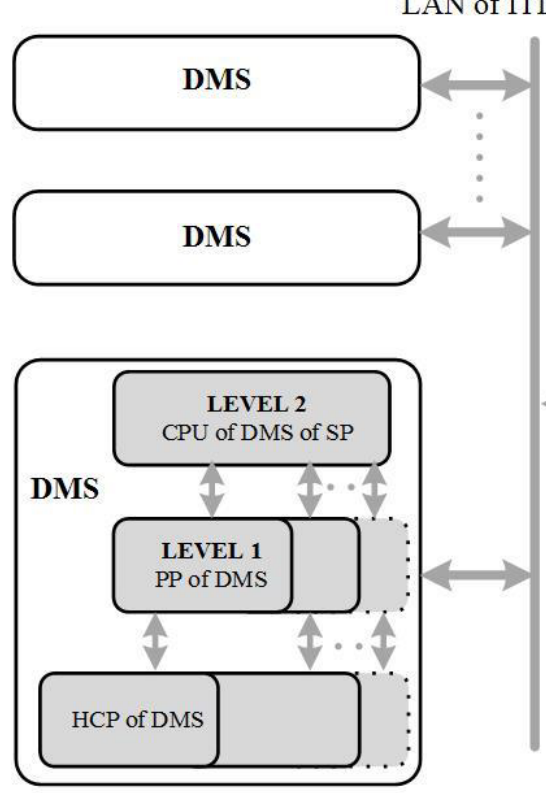

DP EPS
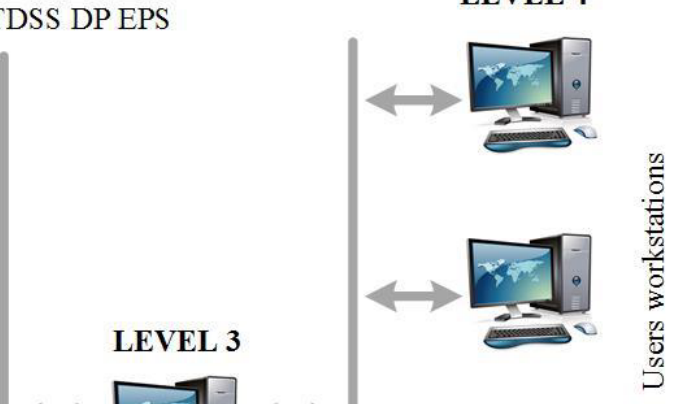

ITDSS DP EPS

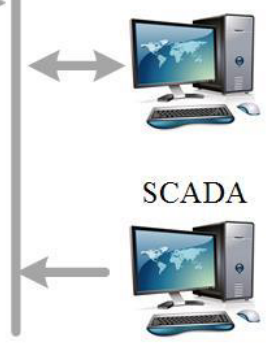

Extranet

Figure 1. The general structure of a multi-level DMS

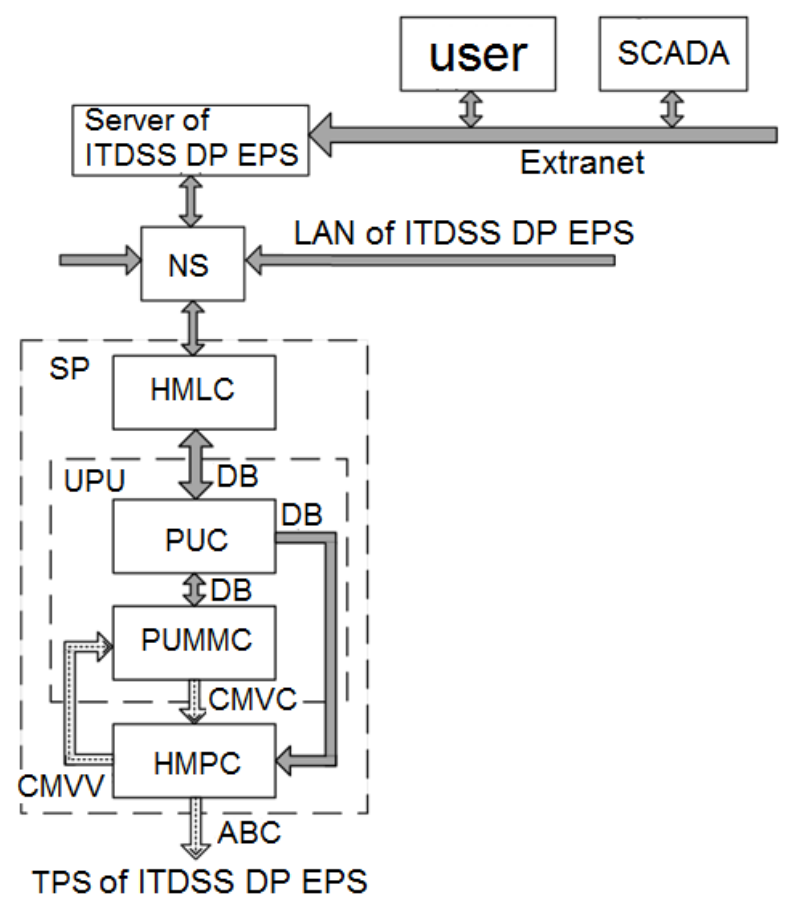

Figure 2. The software and hardware implementation of ITDSS DP EPS 
Indicated in Fig. 1 four levels show hierarchy and structure as in real IEPS. Each level is characterized by the appropriate functionality and reasonable time for performing these functions. The first level of information and control functions, related with digitization, conversion, reporting and implementation of various switching, automatic process's control, is realized by the peripheral processors (PP) of microprocessor units (MPU): processors of analog-digital conversion (PADC), processors of controlled digital switches (processors of commutation - PC), including unipolar and other specialized processors that provide functional control of parameters, states of the simulated equipment and necessary time which is required for these functions execution. All PP of MPU of each hybrid co-processor (HCP) of specialized processor (SP) are interconnected with the second central processing unit (CPU) of MPU. By means of PP and local area network (LAN) informational interaction between CPU and Server of ITDSS DP EPS, including broadcast of database parameters values of the simulated equipment and EPS as a whole from the Server to PP of MPU and to digitalto-analog converters of HCP of all SP of ITDSS DP EPS, is realized. In the same way, transmission of simulation results from SP to Server and then to the user workstation is done. In addition, the local protection, automation and automatic control systems are simulated in CPU. The dynamics of its operation is provided by the time of their execution in the CPU of MPU of SP.

\section{Conclusions}

In conclusion, we would like to note that testing of all components of the ITS DPES had been completed successfully. The system is mounted and ready for experimental researches. Upon completion of the work it will be created the unique tool for operational, including real-time, guaranteed, safe and efficient making of decision for dispatching personnel of IEPS. Due to it the number of accidents in the IEPS will be reduced and energy security of consumers will be ensured.

This work was supported by Russian Federal Target Program "Research and Development in Priority Fields for the Development of Russian Scientific and Technological Complex for 2014-2020" (Grant agreement № 14.575.21.0104 from 28.11.2015, unique identifier of the project RFMEFI57514X0104).

\section{References}

1. A. Naumann, I. Bielchev, N. Voropai, Z. Styczynski, Control Engineering Practice, 25 (1), 102 (2014).

2. S. A. Eroshenko, A. I. Khalyasmaa, S. A. Dmitriev, A. V. Pazderin, A. A. Karpenko, ICPEC, 717 (2013).

3. Smart grids observatory [Online] http://ses.jrc.ec.europa.eu/smart-grids-observatory.

4. Smart grid investment grant program [Online] https://www.smartgrid.gov/recovery act/overview/smart grid investment_grant program.

5. J. Secretarev, A. Zhdanovich, S. Mitrofanov, IFOST, 2, 605 (2013).

6. L. Zhuangzhi, N. Dongxiao, Y. Xusheng, S. Wanxing, Information and Automation, 412 (2009).

7. R. A. Ufa, V. A. Sulaymanova, A. S. Gusev, MATEC Web of Conferences, 19 (2014).

8. Yu. S. Borovikov, A. S. Gusev, A. O. Sulaymanov, R. A. Ufa, IOP Conference Series: Materials Science and Engineering, 66 (2014).

9. Yu. Borovikov, A. Prokhorov, M. Andreev, IFOST, 2, 609 (2012). 